\title{
Assessment of Voltage Dips based on Field Measurements in MV Networks
}

\author{
L.E. Weldemariam, F. Papathanasiou, V. Cuk, J.F.G. Cobben, and W.L. Kling \\ Department of Electrical Engineering \\ Eindhoven University of Technology \\ P.O. Box 513, $5600 \mathrm{MB}$, Eindhoven, The Netherlands \\ L.e.weldemariam@tue.nl
}

\begin{abstract}
Voltage dip is considered as the PQ problem related to the highest financial losses for the customers. It occurs unpredictably mainly due to short-circuit faults in the networks. Network operators and customers want to know more about the occurrence and the potential impact of voltage dips. PQ monitoring is an essential tool for the assessment of voltage dips and it can ultimately contribute to the regulation of voltage dip events. In this paper, firstly the methods of voltage monitoring and voltage dips characterization are discussed. Voltage dip indices of six substations are presented in order to assess the occurrence, type and severity of dips that occur in typical Dutch MV networks. Finally, an approach, which is based on the disrupted loads because of a voltage dip event, is introduced and applied to the data at one substation in order to assess the quality of supply voltage and its implications for the end-users.
\end{abstract}

Index Terms-- assessment of voltage dips, dip monitoring, reporting dips, transfer of dips, voltages dip indices

\section{INTRODUCTION}

There is a continuously growing interest in power quality which is a topic of great importance for future smart grids. Power quality is a great challenge for the network operators who aim to deliver high quality of supply to the customers in a cost effective way. Voltage quality constitutes a main aspect of the power quality domain. Voltage dip is a very important power quality disturbance which is defined as the temporary reduction of the rms voltage at a point in the electrical supply system below a specified start threshold followed by its quick recovery [1]. It can be caused by short-duration increases in current magnitude due to starting of induction motors, switching of heavy loads mostly during reconnection or energizing of transformers, but mainly due to short-circuit faults occurring in the network [2].

The voltage dips have attracted a lot of interest due to the potential negative impact on the equipment of the customers connected to the network causing significant financial losses and technical inconveniences [3], [4]. The modern equipment that is connected to the network is more sensitive to power quality disturbances [4] and the customers are more interested in high quality of supply, as their processes are directly affected by the disturbances in the network. Additionally, there is a growing interest to standardize the several PQ phenomena and define the performance criteria. The regulator bodies need to create a quality framework in terms of power quality indices, and setting the limit for dips is one of the PQ challenges where many countries are striving for. To improve the voltage quality of the network, the grid operator needs more information about the PQ in the network over a longer time period. This has favoured the development and installation of several monitoring devices and extensive measurements that have taken place during the last decades. The significant amount of data obtained from monitors can be analysed to provide better information about the occurrence, type and severity of dips, and this can contribute to the efforts for regulation of voltage dip events.

Several parameters, like the topology of the electrical network, the network impedance at the customer's terminal and the short-circuit power at the point of connection affect the impact of voltage dips on end-users. At present, PQ monitors are mostly meant to measure phase voltages [5], and detected dips have been published in several reports [5], [6]. Different methods of reporting and comparing event indices, site indices and system indices are explained in [5], [6]. The SEMI [7] and ICIT [8] curves have also been used together with the standardized dip table [1] to assess the severity of voltage dips on customer devices. However, the impact of voltage dips to end-users varies depending on the origin and type of dips [5], [9] and also on the type of equipment and processes [5], [10], [11].

In the first place, defining all types of dips in terms of two specific dimensions (magnitude and duration) may lead to substantial loss of information and misinterpretation in statistical reports. For instance, a voltage dip in one phase is less severe for the end-user than an equivalent voltage dip in all the three phases, which is not the case according to the definition in the standards. Different types of dips in the high voltage levels are transferred through different types of transformers leading to different impacts to the end-users. Because extreme weather conditions or a failed attempt of automatic re-closure and re-energizing of a faulted line section, multiple events can occur during a short time interval. Treating these events independently could be useful as a prediction measure of future faults that if appropriate measures are not taken (e.g. replacement of defect component) will lead to persistent faults with potential negative impacts. But such faults are of very short duration which disappear before the protection systems intervene. They may not interrupt the electricity supply and not cause harmful voltage dips. Thus counting these dips separately could overestimate the amount of dips and may lead to misleading conclusions about the impact of the dips to the end-users.

In this paper, a four-year field measurement dip data set of 
the Dutch MV networks is used. After discussion on the methods of voltage dip monitoring and characterization, voltage dip indices of six substations are presented to assess the occurrence, type and severity of dips which are detected in the Dutch MV networks. To assess the impact of voltage dips on end-users, different approaches are explained in this paper. Finally, the influence of the type, magnitude and duration of the dip on the change of the load after the dip event is discussed based on the short-term voltage and active power data obtained from the PQ monitor. This approach provides an insight about interrupted feeder(s) and/or disconnected load(s) because of faults. Moreover, it gives an indication of the end-users immunity to voltage dips, which is presently not well known and needs to be defined in order to set limits for voltage dips in grid codes.

\section{Methodology}

The results presented in this paper are based on a four-year field measurement dip data set of the Dutch MV networks. The four-year dips from six substations were monitored during 2010-2013.

\section{A. Monitoring of Voltage Dips}

Voltage monitoring is an essential step in order to evaluate the voltage dips performance of a network. The voltage monitoring can be a part of PQ measurements which can be integrated into a multifunctional (protection, control, monitoring) system. In the Dutch MV networks, the PQ monitors are installed at the main busbar which is next to the HV/MV transformer- the most optimal location for monitoring voltage dips [12]. Such a system is the so called SASensor system which is implemented in several substations in the Netherlands and results in a significant amount of data acquisition [13]. A simplified network representation of is shown in Figure 1.

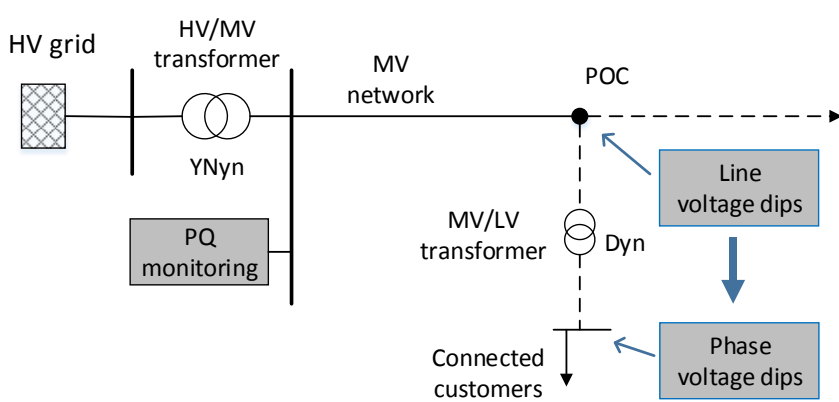

Figure 1. Simplified representation of an electrical network

With SASensors, the voltage measured during the voltage dip event can be recorded as digitalized waveform of high sampling frequency. In order to obtain voltage indices, the rms value of the voltage should be estimated as a function of time. The international standard [14] provides the method of calculating the rms voltage values expressed by (1), where $N$ is the length of the sliding window, $k$ is the index of the sampled voltage value and $u_{i}$ is the value of the instant voltage.

$$
\operatorname{Vrms}(k)=\sqrt{\frac{1}{N} \sum_{i=k-N+1}^{i=k} u_{i}^{2}}
$$

As an example, a high frequency sampled waveform of a voltage dip event recorded by a monitor and its corresponding calculated rms voltage waveform is shown in Figure 2. From the rms voltage waveform, the two indices (magnitude and duration) of each event is calculated and compared with the definition of a voltage dip.

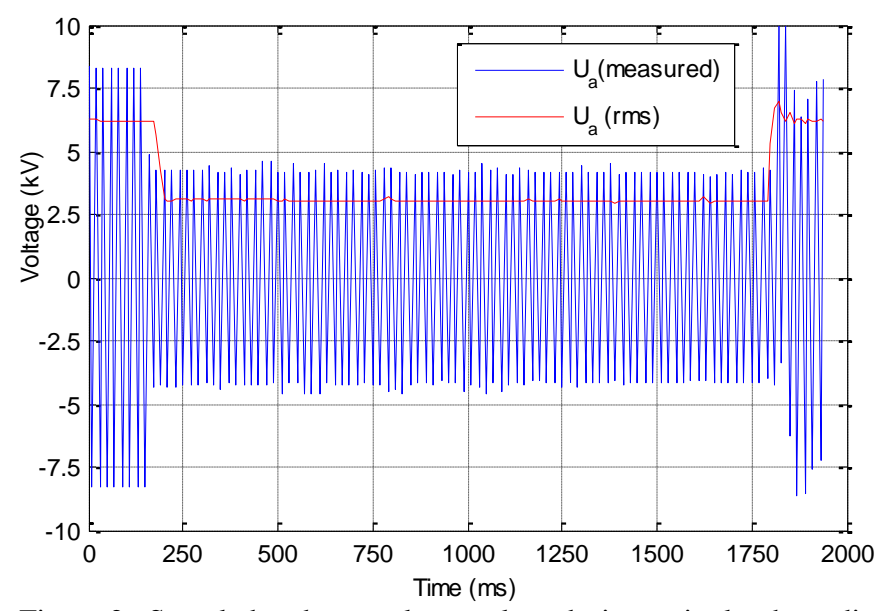

Figure 2. Sampled and rms voltage values during a single-phase dip event

\section{B. Characterization of Voltage Dips}

The characterization of voltage dips depends on the phase voltage measured by the monitors. The voltage dip data includes dips due to different types of faults. For statistical purpose, single event dips can be characterized based on two-dimensions (magnitude and duration). In this work, voltage dips are quantified to the number of phase voltages that drop below the dip threshold value. Based on this, voltage dips are characterized as single-phase, two-phase and three-phase dips. In the Dutch MV networks, very short dips (similar to Figure 3) which probably disappear before the protection system intervenes are found to be common. Although they do not interrupt the electric supply and do not cause harmful voltage dips, they could develop into a persistent single-phase or multiple-phase fault which will ultimately cause interruption of supply and severe dips. Although there no standardized aggregation methods yet (but many suggestions), multiple dip events during a short time interval are aggregated and considered as a single dip event in this paper. The event is with a duration of the sum

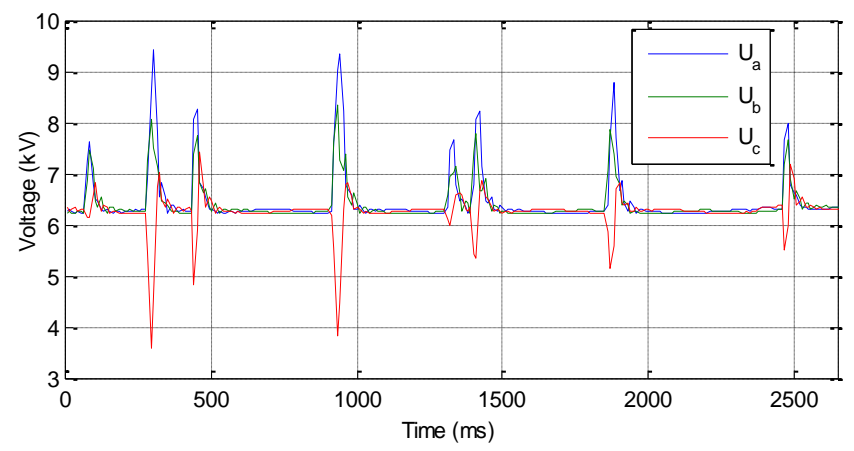

Figure 3. Multiple dip events during a short-time interval 
of the durations of the multiple voltage dip events, and a magnitude equal to the lowest rms value of the voltage during the event.

\section{Assessment of Voltage Dips}

The assessment of voltage dips starts with event indices of each site which are aggregated to constitute system indices following the algorithm shown in Figure 4.

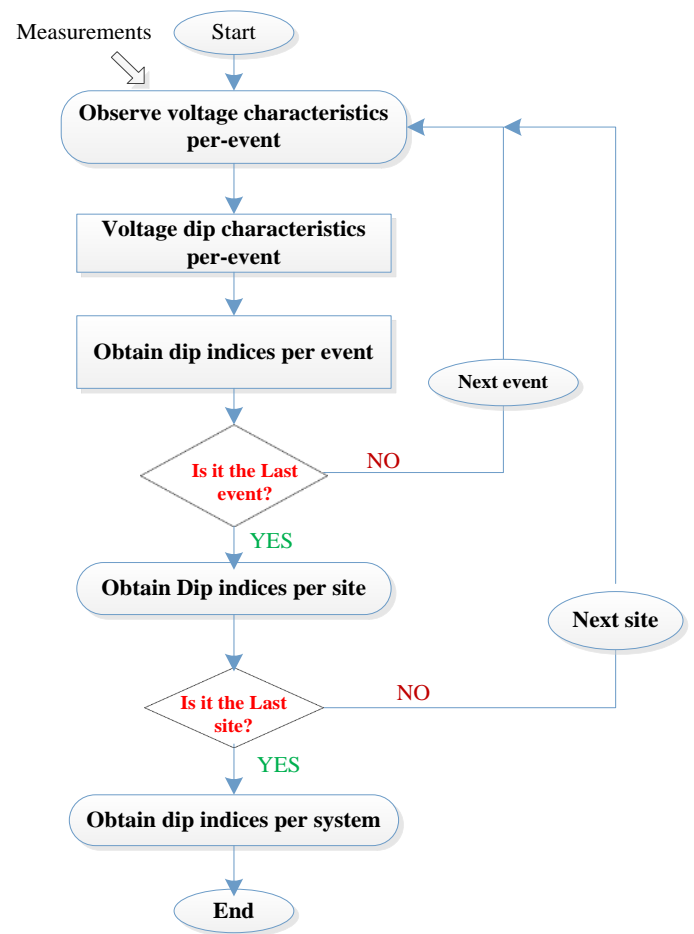

Figure 4. Algorithm followed to calculate voltage dips of six sites during four years

In this paper, two approaches of voltage dip assessments are discussed while considering the quality of the network and impact of dips on end-users.

\section{1) Assessment of dips based on line voltages}

In the Dutch MV networks, the measurement devices are mostly installed at the HV/MV substation and monitor the set of the three phase voltages (phase-to-ground or phase-toneutral voltages). However, sensitive equipment of endusers, which are far away from the monitoring locations and are connected to the network through Dyn transformers, are essentially affected by the line (phase-to-phase) voltages of the MV network. To assess the quality of the networks and sensitivity of customer equipment, the line voltage dips at the MV networks are derived from the phase voltage measurement data, which is recorded by the monitors, using (2).

$$
\begin{aligned}
& V_{a b}(t)=V_{a}(t)-V_{b}(t) \\
& V_{b c}(t)=V_{b}(t)-V_{c}(t) \\
& V_{c a}(t)=V_{c}(t)-V_{a}(t)
\end{aligned}
$$

\section{2) Assessment of dips based loss of load}

During the dip events, high short-circuit currents are developed and loads might be switched off. Based on the active powers measured by the monitor before, during and after the event, an approach of assessment of different types of dips is performed to get an indication about the impact of the dip events.

\section{RESUlts AND DiscuSSION}

\section{A. Transfer of Voltage Dips}

Voltage dips originating in the high voltage levels are affected by transformers before reaching the end-users. To get an insight about how dips affect customers at the LV, the phase and line voltages at the MV network of single-phase and two-phase faults are presented in Figure 5 and Figure 6.
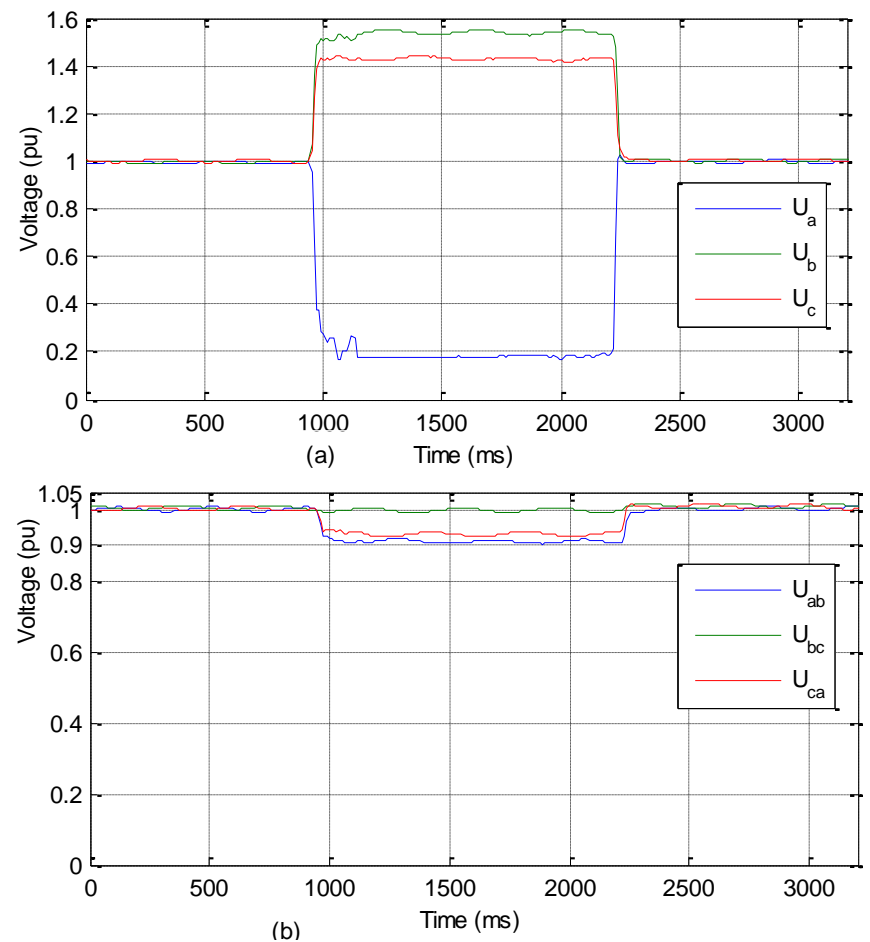

Figure 5. A single-phase dip at the MV network- (a) phase voltage dip, (b) line voltage dip

As it can be seen from Figure 5, a single-phase dip in the MV network (Figure 5 (a)) is not noticed as a line voltage dip (Figure 5 (b)), and thus it does not propagate to the LV network. Whereas, multi-phase dips in the MV network are transferred to the LV network, and this is the case shown in Figure 6.

\section{B. Voltage Dip Indices}

Sampled voltage dip data from the SASensors is processed to obtain the voltage-duration dimensions of each dip events. All single event indices are added up constituting the site indices. In this paper, system indices of six MV substations (sites) monitored for four years are presented in Figure 7. 

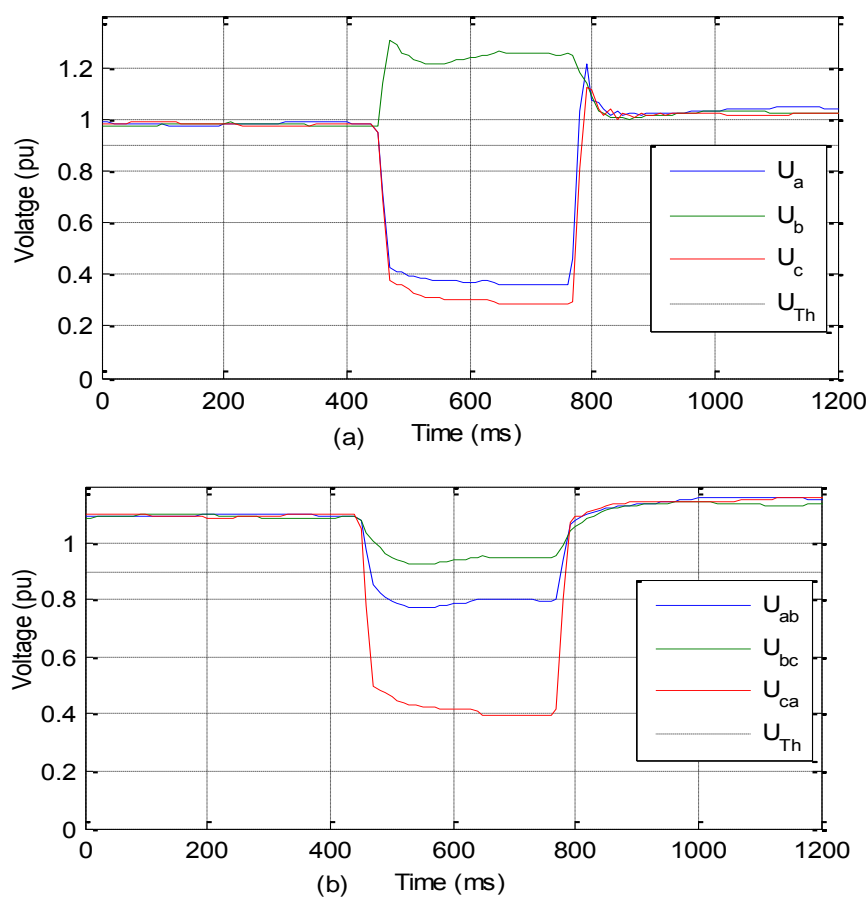

Figure 6. A two-phase dip at the MV network- (a) phase voltage dip,

(b) line voltage dip

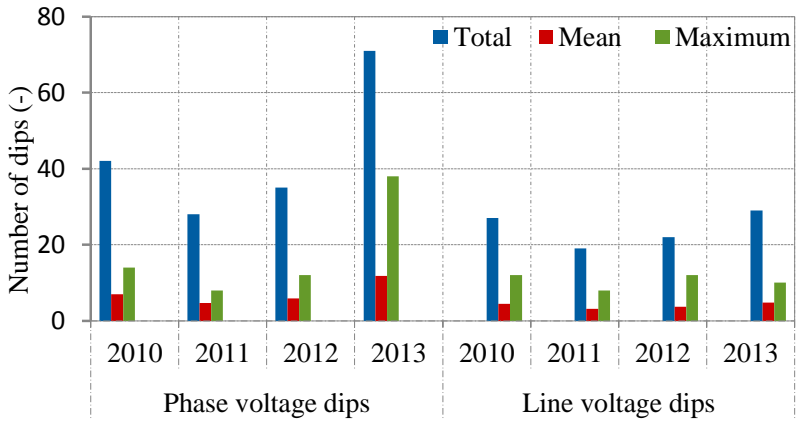

Figure 7. Total, mean and maximum number of phase and line voltage dips recorded at the six MV substations from 2010 to 2013

As it can be seen from Figure 7, the stochastic and random nature of the voltage dips is observed. The occurrence of dips is not constant, not easy to predict and a great fluctuation regarding the yearly number of dips is noticed. Besides, the number of phase voltage dips is significantly greater than the number of line voltage dips. Based on the four-year data set from six MV substations, about 64\% the dips are due to single-phase faults. Two-phase and threephase faults constitute about $22 \%$ and $14 \%$ respectively. On average, about 7.3 phase dips and 4 line dips per year are recorded.

\section{Impact of Voltage Dips on End-users}

The typical two-dimension (magnitude and duration) characterization of voltage dips that is used in most voltage dips statistic reports may lead to substantial loss of information and misinterpretation of the voltage dips data. Based on voltage and active power measurements, a new approach of getting insights about the impact of dips to endusers is introduced in this paper. An example of load loss following a voltage dip event is presented in Figure 8.

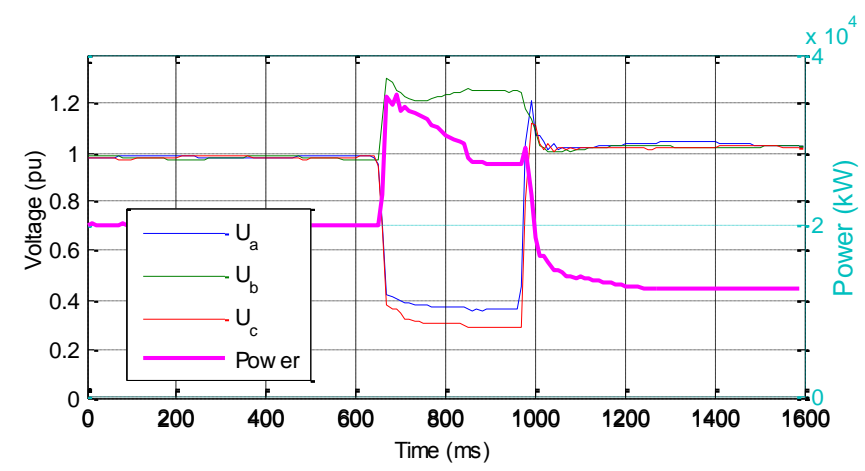

Figure 8. Active power measured during a fault (voltage dip) event in the network

The active power curve in Figure 8 corresponds to a twophase dip event. When the dip occurred, the short-circuit power increased which resulted in the interruption of a feeder and loss of loads. When the fault is cleared and the voltage is recovered, the active power curve is reduced to a new steady state value with respect to the pre-fault active power. This typical example (Figure 8) depicts the reduction of supply power by almost 7MW compared to the pre-fault condition. The assessment of dips based on this approach may provide better insight about interrupted feeder(s) and/or disconnected load(s) because of faults. Moreover, it gives an indication about the sensitivity of end-users to voltage dips.

Using the four-year data set for one MV substation (Nijkerk), classification of load losses are compared with the severity of line dips as in Figure 9. Also, comparisons of load losses for different dip types are presented in Figure 10.

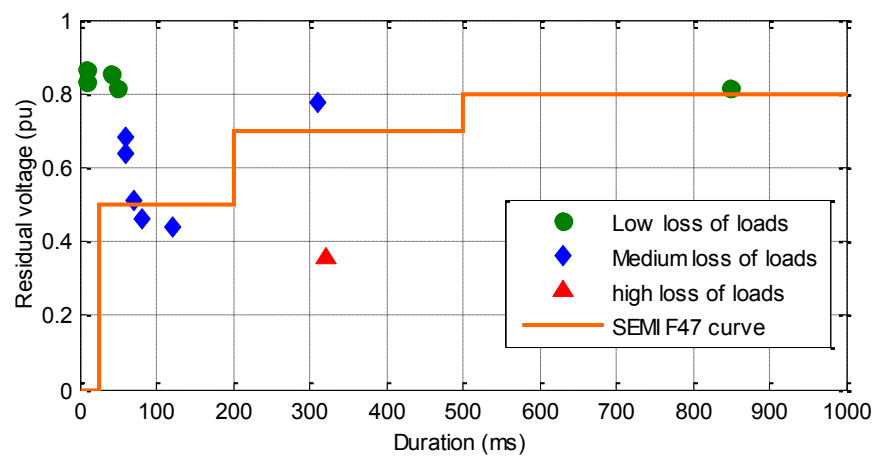

Figure 9. Load losses compared with severity of line voltage dips for Nijkerk MV network during 2010-2013

Figure 9 shows the correlation of line voltage dips, which were recorded in the Nijkerk MV network, with load losses. This analysis is based on a custom classification that considers the total loss of load in all feeders lower than 5\% of the pre-fault situation as low load losses, losses lower than $30 \%$ as medium load losses and losses greater than $30 \%$ as high load losses. It can be noticed that deeper dips are mostly correlated with higher losses of loads. The line voltage dips are plotted in the figure along with the SEMI F47 capability curve. The graph indicates that the line voltage dips that violate the immunity standards set by the curve correspond to considerable load losses. On the other hand, shallow line dips of short duration do not correspond to severe losses of loads. 


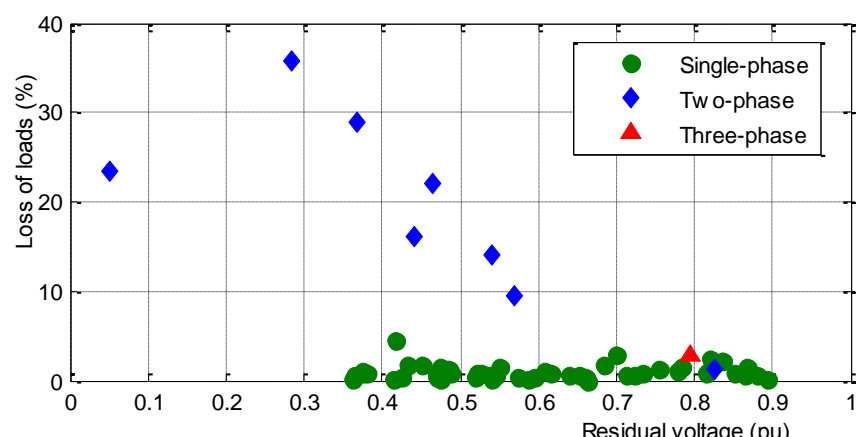

(a)

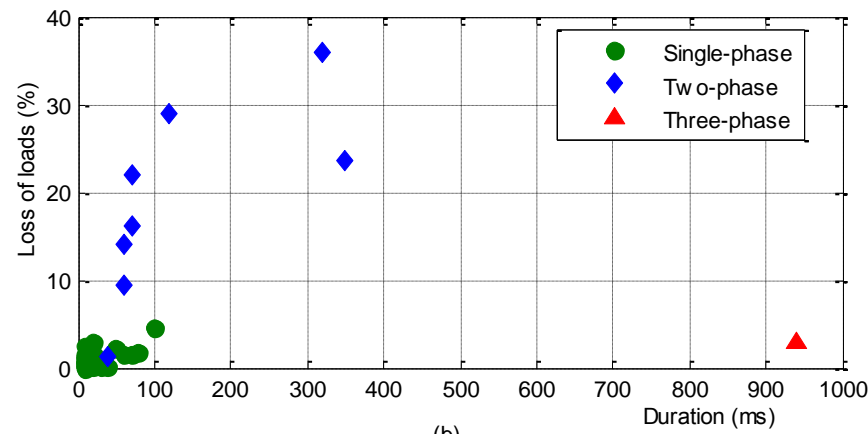

Figure 10. Three-phase classification of dips and losses of loads for Nijkerk MV network during 2010-2013

The correlation between the classification and loss of loads indicates that only two-phase voltage dips are related to significant load losses in the case of the Nijkerk MV network. With the two-phase dips, Figure 10(a) shows that the loss of load generally decreases as the residual voltage of the dip events increase. Whereas single-phase dips show minimum loss of load regardless of the magnitude of the remaining voltage of the dip events. Figure 10(b) also shows that the loss of load with two-phase dip events increases with the duration while the single-phase dips occur for a short duration with minimum load loss.

\section{Conclusion}

With the extensive amount of data acquisition obtained from the PQ monitoring devices, it is possible to adopt common and reliable methods of monitoring and characterizing voltage dips. Special events, such as sequences of multiple dips during a short period of time, should be treated with a standardized method in order to avoid possible misinterpretation of the voltage dips data.

Voltage dip reports based on the monitoring of phase voltages may not provide sufficient information about voltage dip indices and their potential negative impact on the installations of the customer. As the end-users are mostly connected to the MV network through Dyn transformers, line voltage dips at the MV network should essentially be considered to assess the impact of the dips to the end-users.

Based on a four-year measurements campaign in six substations of the Dutch MV networks, a lower number of line dips $(\sim 4)$ is detected compared to the equivalent number of phase voltage dips $(\sim 7.3)$. Moreover, the results of the field measurements analysis indicate that the great majority $(64 \%)$ of voltage dips occur only in one out of the three phase voltages (single-phase voltage dips). This kind of dips is generally not expected to have a severe impact on the end-users.

To assess the impact of voltage dips, two methods are used in this paper - based on the evaluation of detected line voltage dips, and the effect of dips on the power measured at the MV busbar. According to the analysis applied to one MV network, the line dips with a more severe magnitude corresponded to higher loss of loads in the network. Furthermore, two-phase dips were correlated to considerable loss of loads, while voltage dips originating from shortduration single-phase self-extinguishing faults do not seem to have any negative effect on the processes of the endusers.

\section{REFERENCES}

[1] Voltage characteristics of electricity supplied by public electricity networks, European standard ( EN 50160-2010), 2010.

[2] Voltage dips and short interruptions on public electric power supply systems with statistical measurement results, International Standard (IEC 61000-2-8), 2002.

[3] J. Targosz and R. Manson, "Leonardo PQ initiative teamEuropean Power Quality Survey Report", November 2008.

[4] S. Bhattacharrya, J.G.F. Cobben and W.L. Kling, "Assessment of Impacts of Voltage Dips for a MV Customer", IEEE Proc. Of $14^{\text {th }}$ International conference on Harmonics and Quality of Power, Bergamon, Italy, Sep. 2010

[5] CIGRE Working Group, "Voltage dip immunity of equipment and installations- Technical Report of GRE/CIRED/UIE Joint Working Group C4.110", April, 2010.

[6] IEEE Guide for Voltage Sag Indice, IEEE Standard (IEEE Std 1564-2014), 2014

[7] SEMI Curve (SEMI F47), "Specifications of Semiconductor Processing Equipment Voltage Sag Immunity", 2006.

[8] "ITIC Curve- Power Acceptability Curve for Information Technology Equipment", Available: http://www.powerqualityworld.com/2011/04/itic-poweracceptability-curve.html. [Accessed: October 2014].

[9] M. Bollen, Understanding Power Quality Problems- Voltage Sags and Interruptions, New york: IEEE Press, 2000.

[10] S. Ž. Djokic', J. V. Milanovic and D. S. Kirschen, "Sensitivity of AC Coil Contactors to Voltage Sags, Short Interruptions, and Under-voltage Transients", IEEE Transaction on Power Delivery, vol. 19, pp. 1299-1307, July 2004.

[11] S. Ž. Djokic', K.Stockman, J. V. Milanovic, J. J. M. Desmet and R. Belmans, "Sensitivity of AC Adjustable Speed Drives to VoltageSags and Short Interruptions", IEEE Transaction on Power Delivery, vol. 20, pp. 494-505, Jan. 2005.

[12] L.E. Weldemariam, V. Cuk, J.F.G. Cobben and W.L. Kling, "Monitoring of Dips in the MV Network for Regulation- A Case Study", IEEE Proc. of 16th International Conference on Harmonics and Quality of Power (ICHQP), Bucharest, Romania, May 2014.

[13] M.J.M. van Riet, F.L. Baldinger, W.M. van Buijtenen, F.J.T van Erp, F. Volberda and F. Provoost, "Alternative approach for a total integrated secondary installation in MV substations covering all possible required actions", CIRED2005, 2005.

[14] Testing and measurement techniques- Power quality measurement, International Standard (IEC 61000-4-30), 2008. 